\title{
Disociación escapulotorácica traumática: Una entidad para tener en cuenta en caso de trauma
}

\author{
Dres. David Gómez A ${ }^{(1)}$, Catalina Wilches $V^{(2)}$.
}

1. Residente de IV año de Radiología e Imágenes Diagnósticas. Fundación Universitaria de Ciencias de la Salud. Colombia.

2. Médica Radióloga. Clínica Reina Sofía. Organización Sanitas Internacional, Colombia.

\section{Traumatic scapulothoracic dissociation: An entity to be considered in case of trauma}

\begin{abstract}
Traumatic scapulothoracic dissociation represents the separation of the scapula from the chest wall. The mechanism of this trauma involves a severe traction applied to the upper member which translates into neurovascular and musculoskeletal injuries secondary to trauma. This lesion often goes unnoticed for appearing in conjunction with major trauma or presenting with bizarre symptoms. On physical examination, patients exhibit swelling of soft tissue around the shoulder and upper limb. Lateral displacement of scapula greater than $1 \mathrm{~cm}$ is the classic radiological finding on thoracic imaging studies. The key is to compare the distance from medial borders of the scapula to midline (dorsal column).
\end{abstract}

Key words: Scapulothoracic dissociation, Subclavian vessels injury.

Resumen: La disociación escapulotorácica traumática como su nombre lo indica, se define como la separación de la escápula de la pared del tórax. El mecanismo de este trauma implica una tracción severa aplicada al miembro superior con lesiones neurovasculares y musculoesqueléticas secundarias.

Esta lesión suele pasar desapercibida por presentarse en conjunto con traumas mayores o cursar con síntomas bizarros. Al examen físico, los pacientes presentan edema de tejidos blandos alrededor del hombro y de la parte superior de la extremidad.

El hallazgo radiológico clásico en la radiografía de tórax es el desplazamiento lateral mayor a $1 \mathrm{~cm}$ de la escápula con respecto al tórax. La clave está en comparar la distancia de los bordes mediales de las escápulas en relación a una línea media en la columna dorsal.

Palabras clave: Disociación escapulotorácica, Lesiones de vasos subclavios.

Gómez $D$, et al. Disociación escapulotorácica traumática: Una entidad para tener en cuenta en caso de trauma. Rev Chil Radiol 2012; 18(4): 163-166.

Correspondencia: catalinawilches@yahoo.com,cwilches30@hotmail.com

Trabajo recibido el 16 de julio de 2012, aceptado para publicación el 24 de octubre de 2012.

\section{Introducción}

La disociación escapulotorácica traumática como su nombre lo indica, se define como la separación de la escápula de la pared del tórax. El mecanismo de este trauma implica una tracción severa aplicada al miembro superior con lesiones neurovasculares y musculoesqueléticas secundarias ${ }^{(1)}$.

Esta lesión suele pasar desapercibida por presentarse en conjunto con traumas mayores o cursar con síntomas bizarros, por lo cual es importante tenerla en mente en casos de trauma, dada su alta morbimortalidad $^{(2)}$.

Dado que nuestro hospital no maneja un volumen importante de trauma, no se pensó en esta entidad en el paciente que vamos a exponer a continuación, sólo hasta que se valoró la causa del fallecimiento, lo cual nos dejó muchas enseñanzas a todo el equipo de Urgencias y a nosotros como radiólogos.

\section{Presentación de caso}

Hombre de 72 años de edad quien presenta trauma en abducción del miembro superior derecho al sujetarse para no caer al frenar de manera abrupta el vehículo de transporte público masivo en el que viajaba.

Como único antecedente refiere diabetes mellitus II controlada.

Al ingresar al servicio de Urgencias acusa dolor torácico y del aspecto proximal del miembro superior derecho, por lo cual le solicitan una radiografía de tórax y de hombro, reportando por el radiólogo de turno un aumento en la amplitud del espacio articular 
acromioclavicular (Figuras 1 y 2 ).

El paciente presenta deterioro de su estado general y aumento del dolor torácico. Se ordena tomografía computada de tórax con medio de contraste endovenoso, en la cual se reporta importante edema de tejidos blandos del hemitórax y del aspecto proximal del miembro superior derecho, aumento en la amplitud del espacio articular acromioclavicular y sugerencia de trombosis de la vena subclavia derecha, por falta de opacificación de la misma con el contraste (Figuras 3-6).

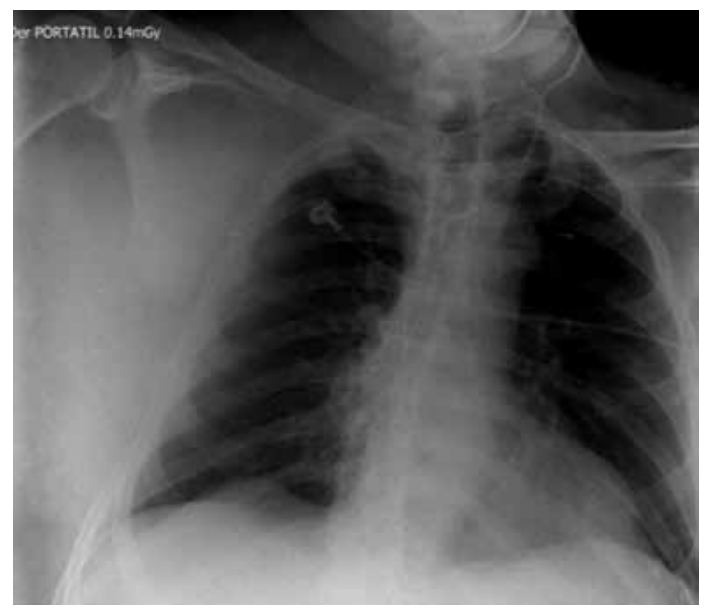

Figura 1. Radiografía anteroposterior y portátil de tórax. Se evidencia desplazamiento escapular lateral derecho, con aumento de la distancia entre el borde medial de la escápula derecha y las apófisis espinosas, mayor a $1 \mathrm{~cm}$ con respeto al contralateral. Aumento en el espesor y la densidad de los tejidos blandos de la pared derecha del tórax y del aspecto proximal del miembro superior derecho.

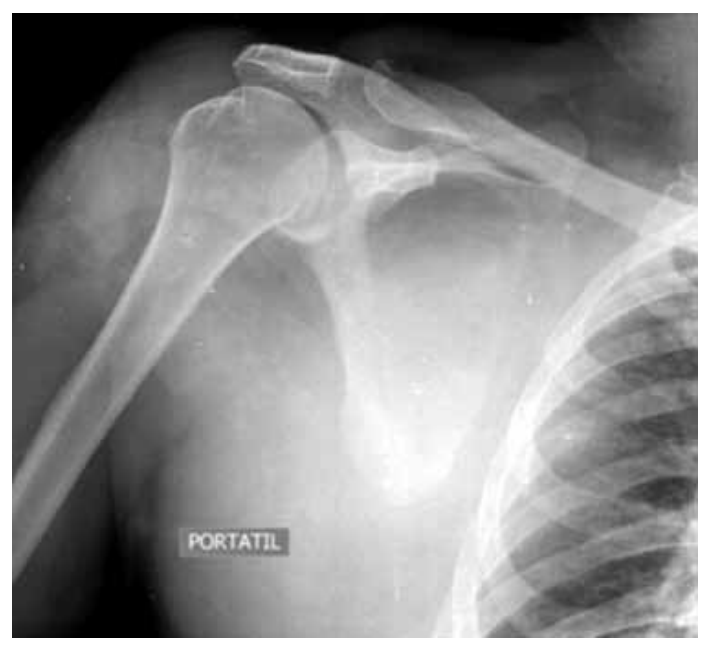

Figura 2. Radiografía anteroposterior y portátil de hombro derecho. Se observan los cambios descritos en la radiografía de tórax y el aumento en el espacio articular acromioclavicular.
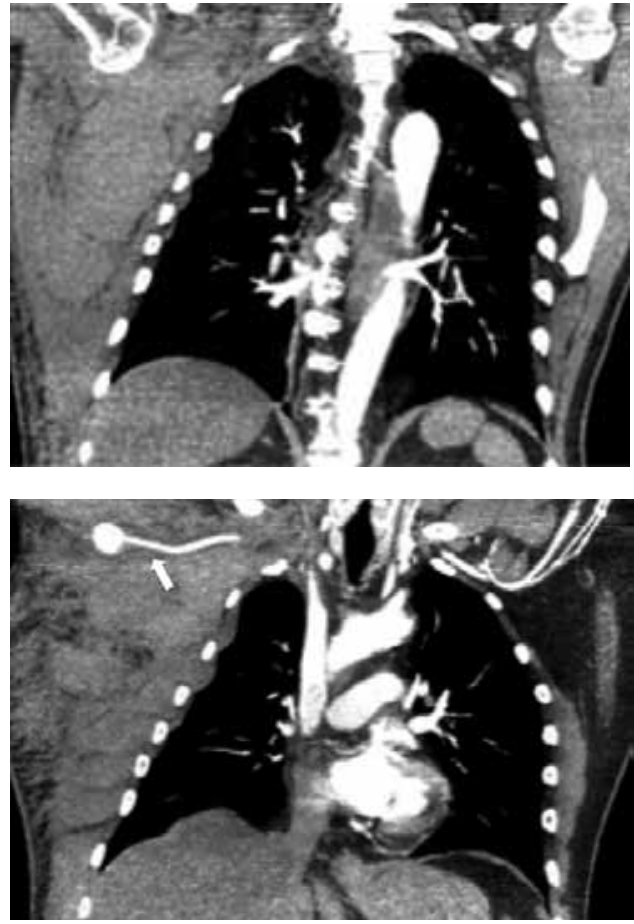

Figura 3-4. Reconstrucciones coronales de tomografía computada de tórax con medio de contraste endovenoso. Se evidencia importante edema de tejidos blandos y hematoma de gran tamaño en la pared del hemitórax derecho y el extremo proximal del miembro superior de este lado. Además se observa ausencia de opacificación de la vena subclavia derecha, por amputación traumática de la misma (flecha).
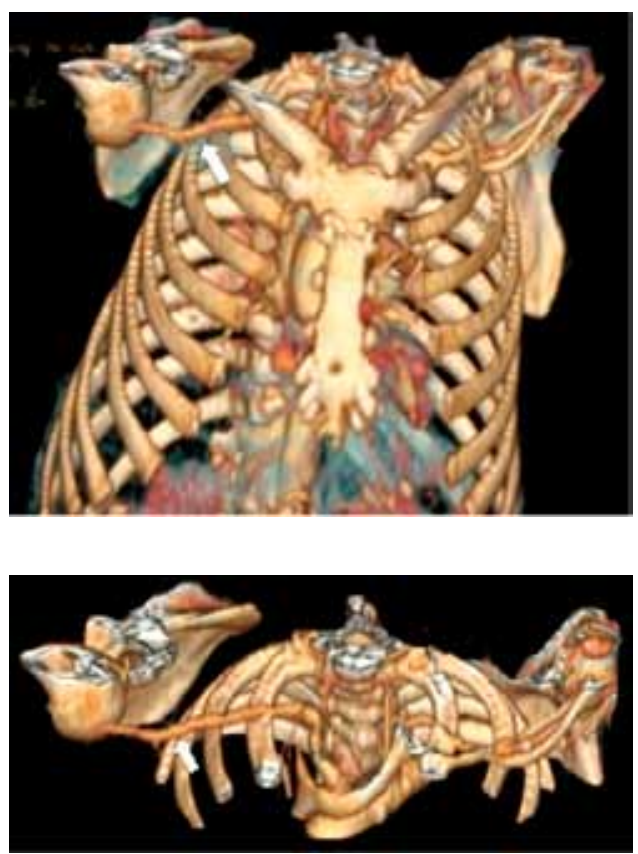

Figura 5-6. Reconstrucciones tridimensionales de tomografía computada de tórax en las que se evidencia la lateralización de la escápula derecha, y la ausencia de visualización de la vena subclavia derecha, por amputación traumática de la misma (flecha). 
El paciente presenta mayor deterioro y muere 17 horas después de su ingreso.

Se valoró de forma interdisciplinaria y retrospectiva el caso de nuestro paciente, revisando la historia clínica y las imágenes diagnósticas. Se llegó a la conclusión que la causa del fallecimiento se debió a una disociación escapulotorácica traumática, que generó un tercer espacio, que llevó a shock hipovolémico, posterior falla multisistémica y finalmente la muerte.

\section{Discusión}

Patogenia. La disociación escapulotorácica traumática es comúnmente el resultado de una fuerza de tracción masiva en la extremidad superior o una fuerza directa contundente ${ }^{(3,4)}$.

El mecanismo clásico consiste en un violento desplazamiento lateral por tracción con desplazamiento de la cintura escapular. La extremidad superior queda fija, mientras que el cuerpo se está moviendo a alta velocidad(2).

Los músculos comprometidos por avulsión total o parcial son el deltoides, pectoral menor, elevador de la escápula, dorsal y trapecio.

El plexo braquial por lo general sufre avulsión proximal y las lesiones de este plexo se producen en el $94 \%$ de los pacientes con secuelas importantes no reversibles ${ }^{(5)}$.

Los vasos subclavios, por lo general la vena, puede ser lesionada en su porción torácica o proximal al tórax y por el daño endoluminal puede producirse compromiso trombótico(2).

Por el desplazamiento traumático de la escápula se puede generar un tercer espacio que permite la formación de un gran hematoma, como el caso de nuestro paciente.

La isquemia es menos frecuente, ya que la extremidad cuenta con una completa red de colaterales que disminuyen esta posibilidad ${ }^{(2)}$.

\section{Diagnóstico Clínico}

Los pacientes presentan edema, hematomas palpables y debilidad en la extremidad. La piel del paciente a menudo está intacta, ocultando la gravedad de la lesión subyacente ${ }^{(6-8)}$.

Las fracturas y las luxaciones por lo general son palpables por lo que un buen examen puede ayudar a sospechar esta entidad ${ }^{(7,8)}$.

Un error común es atribuir la ausencia de pulso a una lesión distal, sin pensar que se trata de compromiso proximal, que amenaza la vida(8).

Es importante tener en cuenta que la ausencia de una lesión neurovascular no excluye el diagnóstico de disociación escapulotorácica, y acerca de esta posibilidad existen varios reportes en la literatura ${ }^{(9-12)}$.

\section{Diagnóstico Radiológico}

En la proyección anteroposterior (AP) de la ra- diografía de tórax puede valorarse el desplazamiento lateral de la escápula y la separación acromioclavicular, así como fracturas de la clavícula ${ }^{(2)}$.

En la radiografía de tórax el desplazamiento escapulotorácico se diagnostica con una diferencia mayor a $1 \mathrm{~cm}$ en la distancia entre el borde medial de la escápula y la apófisis espinosa en el lado afectado al compararlo con el contralateral, como en el caso de nuestro paciente ${ }^{(2,6)}$ (esquema 1).

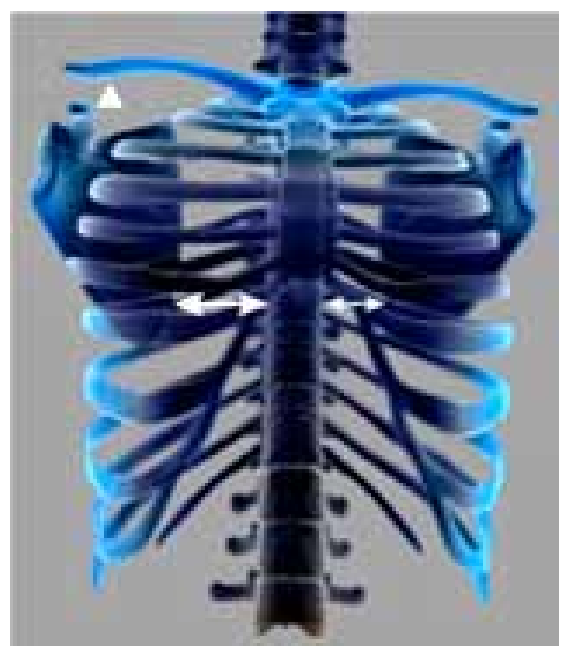

Esquema 1. Esquema de la medición del desplazamiento escapulotorácico en la radiografía de tórax (flecha). Elevación asociada de la clavícula (cabeza de flecha).

En la tomografía computada con reconstrucciones multiplanares se puede evidenciar la pérdida de las relaciones articulares y la presencia de edema y hematomas en los tejidos blandos, hallazgos visualizados en nuestro paciente ${ }^{(13,14)}$.

La angiografía por tomografía computada o convencional sirven para confirmar la amputación de los vasos subclavios ${ }^{(13)}$. En nuestro caso se reportó la ausencia de flujo en la vena subclavia derecha, y se interpretó inicialmente como posible trombosis y de manera retrospectiva se concluyó que se trataba de amputación traumática de este vena.

La ecografía Doppler de miembros superiores suele ser difícil técnicamente por asociarse la disociación generalmente a importante edema de tejidos blandos, pero puede ser útil para evidenciar la ausencia de flujo en los vasos subclavios.

La resonancia magnética y la mielografía por tomografía pueden ser útiles para detectar las avulsiones radiculares cervicales con sensibilidad del $93 \%{ }^{(15)}$.

\section{Clasificación}

Varios esquemas de clasificación han sido descritos, pero Damschen y colaboradores propusieron 
un sistema lógico y útil que reconoce la importante variabilidad en la presentación clínica ${ }^{(5)}$. Su esquema de clasificación describe el grado de lesión basado en los hallazgos clínicos (Tabla I).

Tabla I. Clasificación para la disociación escapulotorácica de Damschen y cols. Se basa en el sistema comprometido.

\begin{tabular}{|cl|} 
Tipo & \multicolumn{1}{c|}{ Lesión } \\
\hline I & Musculoesquelética \\
IIA & Musculoesquelética - vascular \\
IIB & Musculoesquelética - neurológico \\
III & Musculoesquelética - neurovascular \\
\hline
\end{tabular}

\section{Conclusión}

La disociación escapulotorácica traumática es una patología poco común consecuencia de un trauma, generalmente de alto impacto generado sobre la extremidad superior, que como su nombre lo indica condiciona separación de la escápula y la pared del tórax, asociada a lesión musculoesquelética y de forma variable compromiso vascular o neurológico.

Es de difícil diagnóstico dada su rara presentación y sus signos y síntomas bizarros, con alta morbimortalidad por lo cual es importante tenerla en cuenta al valorar un paciente con este tipo de trauma en las extremidades superiores, para buscar y reportar de forma clara y rápida el probable compromiso vascular y neurológico, y guiar al médico tratante en el servicio de Urgencias en cuanto a las imágenes diagnósticas que debe solicitar con este fin.

\section{Bibliografía}

1. Jbara M, Bajwa J, Hahn B. Scapulothoracic dissociation. J Emerg Med. 2012 Mar 8. [Epub ahead of print] doi: 10.1016/j.jemermed.2012.01.017
2. Althausen PL, Lee MA, Finkemeier CG. Scapulothoracic dissociation: diagnosis and treatment. Clin Orthop Relat Res. 2003 Nov; (416): 237-244.

3. Lange $\mathrm{RH}$, Noel $\mathrm{SH}$ : Traumatic lateral scapular displacement: An expanded spectrum of associated neurovascular injury. J Orthop Trauma 1983; 7: 361-366.

4. Lavelle WM, Uhl R. Scapulothoracic dissociation. Orthopaedics 2010; 33: 417e21.

5. Damschen DD, Cogbill TH, Siegel MJ: Scapulo- thoracic dissociation caused by blunt trauma. J Trauma 1997; 42: 537-540.

6. Oreck SL, Burgess A, Levine AM: Traumatic lateral displacement of the scapula: A radiographic sign of neurovascular disruption. J Bone Joint Surg 1984; 66A: 758-763.

7. Zelle BA, Pape HC, Gerich TH, Garapati R, Ceylan B, Krettek C. Functional outcome following scapulothoracic dissociation. J Bone Joint Surg 2004; 86-A: 2-8.

8. Sen RK, Prasad G, Aggarwal S. Scapulothoracic dissociation: level of vascular insult, an indirect prognostic indicator for the final outcome? Acta Orthop. Belg 2009; 75: 14-18.

9. Leech C. Scapulothoracic dissociation. Emerg Med J 2012; 29: 305.

10. Kelbel JM, Jardon OM, Huurman WW. Scapulothoracic dissociation. A case report. Clin Orthop 1986; 209: 210-214.

11. Nagi ON, Dhillon MS. Traumatic scapulothoracic dissociation. A case report. Arch Orthop Trauma Surg 1992; 111: 348-349.

12. Tuzuner S, Yanat AN, Urguden M, Ozkaynak C.Scapulothoracic dissociation: a case report. Isr J Med Sci 1995; 32: 70-74.

13. Kelbel JM, Jardon OM, Huurman WW: Scapulothoracic dissociation: A case report. Clin Orthop 1986; 209: 210-214.

14. Witz M, Korzets Z, Lehmann J. Traumatic scapulothoracic dissociation. J Cardiovasc Surg (Torino) 2000; 41: 927-929.

15. Doi K, Otsuka K, Okamoto Y, et al: Cervical nerve root avulsion in brachial plexus injuries: Magnetic resonance imaging classification and comparison with myelography and computerized tomography myelography. J Neurosurg 2002; 96: 277-284. 